\title{
Storing light with subradiant correlations in arrays of atoms
}

\author{
G. Facchinetti, ${ }^{1,2}$ S. D. Jenkins, ${ }^{1}$ and J. Ruostekoski ${ }^{1}$ \\ ${ }^{1}$ Mathematical Sciences, University of Southampton, Southampton SO17 1BJ, United Kingdom \\ ${ }^{2}$ École Normale Supérieure de Cachan, 61 avenue du Président Wilson, 94235 Cachan, France
}

(Dated: October 13, 2016)

\begin{abstract}
We show how strong light-mediated resonant dipole-dipole interactions between atoms can be utilized in a control and storage of light. The method is based on a high-fidelity preparation of a collective atomic excitation in a single correlated subradiant eigenmode in a lattice. We demonstrate how a simple phenomenological model captures the qualitative features of the dynamics and sharp transmission resonances that may find applications in sensing.
\end{abstract}

Resonant emitters play a key role in optical devices for classical and quantum technologies. Atoms have particular advantages because of an excellent isolation from environmental noise with well-specified resonance frequencies and no absorption due to nonradiative losses. At high densities, however, they exhibit strong light-mediated resonant dipole-dipole (DD) interactions that can lead to uncontrolled and unwanted phenomena, such as resonance broadening, shifts and dephasing. According to common wisdom, these are considered as a design limitation in quantum and classical light technologies, e.g., in quantum metrology [1, 2], sensing [3], information processing [4, in the storage of light and in the implementations of quantum memories [5] 8]. DD interactions also receive significant attention, e.g., in Rydberg gases [9 13. Here we show how strong radiative interactions can be harnessed in engineering long-living collective excitations that open up avenues for utilizing resonant DD interactions in the control and storage of light, and in sensing. Our protocol is based on controlled preparation of large, many-atom subradiant excitations, where the light-mediated interactions between the atoms strongly suppress radiative losses.

Superradiance 14 where the emission of light is coherently enhanced in an ensemble of emitters has continued to attract considerable interest [15] with the recent experiments focusing on light in confined geometries [16], weak excitation regime [17-19, and the related shifts of the resonance frequencies 20 24. Its counterpart, subradiance, describes coherently suppressed emission due to a weak coupling to the radiative vacuum. Because of the weak coupling, subradiant states are challenging to excite and have experimentally proved elusive. In atomic and molecular systems subradiance has been observed in pairs of trapped ions [25] and molecules [26], as well as in weakly bound ultracold molecular states [27, 28. In a large atom cloud a subradiant decay was recently observed in the long tails of a radiative decay distribution [29] that indicated a small fraction of the atoms exhibiting a suppressed emission.

In our model, an incident light excites a collective atomic state that exhibits a significant radiative vacuum coupling. The excitation is then transferred to a radia-

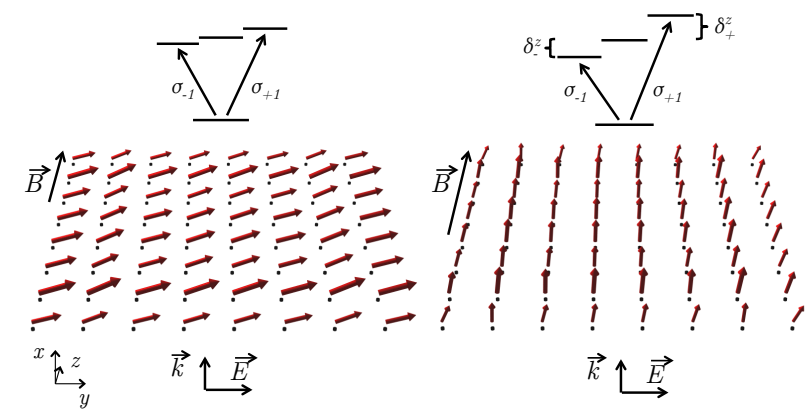

FIG. 1. Schematic illustration and numerically calculated response. The atoms (one per site) are confined in a small square $2 \mathrm{D} 8 \times 8$ array on the $y z$ plane. The linearly polarized (along $y$ ) incident light propagates along the positive $x$ direction driving the $\left|J=0, m_{J}=0\right\rangle \rightarrow\left|J^{\prime}=1, m_{J}= \pm 1\right\rangle$ transitions. The arrows represent the numerically calculated steady-state atomic dipoles at each site. A real or synthetic magnetic field along the $z$ axis induces Zeeman shifts, effectively rotating the dipoles around the $z$ axis. For $\left(\delta_{+}^{z}, \delta_{-}^{z}\right)=(0.1,0.3) \gamma$ (left panel) this rotation is small, but for $\left(\delta_{+}^{z}, \delta_{-}^{z}\right)=(0.45,1.75) \gamma$ (driven at the resonance of the subradiant mode; right panel), the dipoles are oriented approximately normal to the lattice, representing a collective excitation eigenmode with a factor of 50 narrowed linewidth.

tively isolated cooperative state. The cold atoms that store the light excitation are confined in a planar lattice, providing a protection against nonradiative losses-that typically are a common hindrance to observation of subradiance. The state transfer is achieved by rotating the collective atomic polarization by an effective magnetic field. Depending on the size of the lattice and the confinement of the atoms, we find substantially suppressed radiative emission where up to $98-99 \%$ of the total excitation is transferred into a single subradiant eigenmode of the interacting multiatom system. The correlated manyatom excitation spatially extends over the entire lattice and is therefore fundamentally different from two-atom subradiant states 25, 27, 28. We develop a simple phenomenological two-mode model that provides an intuitive description of the light storage dynamics, and qualitatively captures the essential features, e.g., of the Fano resonance of the forward-scattered light.

We consider a tightly-confined square planar array of atoms (e.g., a 2D optical lattice) with one atom per site 
(Fig. 1). The light-induced radiative DD interactions lead to collective behavior of the atoms in the lattice that is dramatically different from the response of an individual, isolated atom 30 34. The atoms are either at fixed positions or we address the position fluctuations using the model of a finite optical lattice with the potential depth $s E_{R}$ in the units of the lattice photon recoil energy $E_{R}$ 35, 36. In the numerics, the lattice spacing $a=0.55 \lambda$, except when specified otherwise. Whenever we consider a finite lattice depth, we take the confinement normal to the lattice $\simeq 0.12 a$. The atoms are illuminated by an incident weak-intensity laser with the amplitude $\mathcal{E}(\mathbf{r})=\mathcal{E}_{0}(y, z) \hat{\mathbf{e}}_{y} \exp (i k x)$, with polarization $\hat{\mathbf{e}}_{y}$ and $\mathcal{E}_{0}(y, z)$ either constant or a Gaussian profile on the $y z$ plane. Here, and in the rest of the paper, all the field amplitudes and the atomic polarization correspond to the slowly varying positive frequency components with oscillations at the laser frequency $\omega$. We consider a nearresonance $J=0 \rightarrow J^{\prime}=1$ atomic transition (e.g. $\mathrm{Yb}, \mathrm{Sr}$ ) and assume a controllable Zeeman level splitting of the $J^{\prime}=1$ manifold. The Zeeman shifts could be induced by magnetic fields or, e.g., by AC Stark shifts [37].

In the numerical simulations we calculate the optical response by evaluating all the multiple scattering events [38, 39] between the atoms in an array. In the limit of low light intensity, for stationary atoms the results are exact [40, 41, and we also include the vacuum fluctuations of the atomic positions in the lowest vibrational level of each lattice site 30. This is done by stochastically sampling the atomic positions at each site in each realization according to the density distribution and then ensemble-averaging the results. At each stochastic run we have the $N$ atoms fixed at positions $\mathbf{r}_{j}$, and we calculate the dipole moment $\mathbf{d}_{j}=\mathcal{D} \sum_{\sigma} \hat{\mathbf{e}}_{\sigma} \mathcal{P}_{\sigma}^{(j)}$ for each atom $j$, where $\mathcal{D}$ denotes the reduced dipole matrix element. Each atom has three polarization amplitude components $\mathcal{P}_{\sigma}^{(j)}$ associated with the unit circular polarization vectors $\hat{\mathbf{e}}_{ \pm 1}=\mp\left(\hat{\mathbf{e}}_{x} \pm i \hat{\mathbf{e}}_{y}\right) / \sqrt{2}$ and $\hat{\mathbf{e}}_{0}=\hat{\mathbf{e}}_{z}$, that are coupled with the transitions $|J=0, m=0\rangle \rightarrow\left|J^{\prime}=1, m=\sigma\right\rangle$.

In the limit of low light intensity, the excited state population of the atoms vanishes and the excitation amplitudes satisfy [30, 41]

$$
\frac{d}{d t} \mathcal{P}_{\sigma}^{(j)}=\left(i \Delta_{\sigma}-\gamma\right) \mathcal{P}_{\sigma}^{(j)}+i \frac{\xi}{\mathcal{D}} \hat{\mathbf{e}}_{\sigma}^{*} \cdot \epsilon_{0} \mathbf{E}_{\mathrm{ext}}\left(\mathbf{r}_{j}\right),
$$

where $\xi=6 \pi \gamma / k^{3}$ and the single-atom Wigner-Weisskopf linewidth $\gamma=\mathcal{D}^{2} k^{3} /\left(6 \pi \hbar \epsilon_{0}\right)$. The detuning from the atomic resonance $\Delta_{\sigma}=\omega-\omega_{\sigma}=\omega-\left(\omega_{0}+\sigma \delta_{\sigma}^{z}\right)$ where $\omega_{0}$ is the resonance frequency of the $|J=0\rangle \leftrightarrow \mid J^{\prime}=$ $1, m=0\rangle$ transition and $\pm \delta_{ \pm}^{z}$ are the shifts of the $m= \pm 1$ levels (Fig. 1). Each amplitude in Eq. (1) is driven by the sum of the incident field and the fields scattered from all the other $N-1$ atoms $\mathbf{E}_{\text {ext }}\left(\mathbf{r}_{j}\right)=\mathcal{E}\left(\mathbf{r}_{j}\right)+\sum_{l \neq j} \mathbf{E}_{S}^{(l)}\left(\mathbf{r}_{j}\right)$. The scattered dipole radiation field from the atom $l$ is $\epsilon_{0} \mathbf{E}_{S}^{(l)}(\mathbf{r})=\mathrm{G}\left(\mathbf{r}-\mathbf{r}_{l}\right) \mathcal{D} \sum_{\sigma} \hat{\mathbf{e}}_{\sigma} \mathcal{P}_{\sigma}^{(l)}$, where $\mathrm{G}$ is the dipole

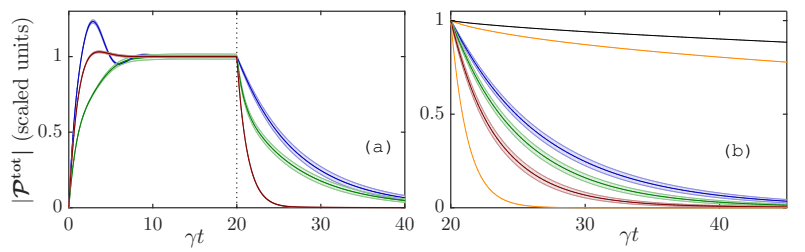

FIG. 2. The dynamics of the total atomic polarization density in the lattice of $20 \times 20$ sites for different Zeeman shifts and lattice heights. (a) Curves from slow to fast decay: $\left(\delta_{+}^{z}, \delta_{-}^{z}, \Delta_{0}\right)=(0,0,0),(0.4,0.6,0) \gamma$, and $(1.1,1.1,0.65) \gamma$ $(s=50)$. At $\gamma t=20$ (when each curve is normalized to one), the Zeeman shifts and the incident light are switched off. When the dipoles are oriented close to the $x$ axis (see Fig. 1 on right), the decay is slow (collective subradiance). For $\delta_{ \pm}^{2}=0$ the dipoles are pointing along the $y$ axis and decay rapidly. An exponential fitting provides decay rates $0.79 \gamma, 0.16 \gamma$, and $0.14 \gamma$. (b) The curves from top: incident Gaussian beam with fixed atomic positions, plane-wave excitation for fixed atomic positions, for lattice with $s=50$, $20,5,\left[\left(\delta_{+}^{z}, \delta_{-}^{z}, \Delta_{0}\right)=(1.1,1.1,0.65) \gamma\right]$, and for fixed atomic positions $\left(\delta_{ \pm}^{z}=\Delta_{0}=0\right)$. The shaded lines around the curves represent the stochastic uncertainties of the excitation amplitudes due to the vacuum fluctuations of the atomic positions.

radiation kernel, such that $\mathbf{E}_{S}^{(l)}(\mathbf{r})$ represents the electric field at $\mathbf{r}$ from a dipole $\mathcal{D} \sum_{\sigma} \hat{\mathbf{e}}_{\sigma} \mathcal{P}_{\sigma}^{(l)}$ residing at $\mathbf{r}_{l}$ [42].

We first consider a single, isolated atom. This is obtained in Eq. (1) by setting $\mathbf{E}_{\text {ext }}\left(\mathbf{r}_{j}\right) \rightarrow \mathcal{E}\left(\mathbf{r}_{j}\right)$. The $y$ polarized light then drives the atomic polarization components $\mathcal{P}_{ \pm 1}^{(j)}$ (Fig. 1). Here we instead write the equations of motion in the Cartesian basis $\mathbf{d}_{j} / \mathcal{D}=\hat{\mathbf{e}}_{x} \mathcal{P}_{x}^{(j)}+$ $\hat{\mathbf{e}}_{y} \mathcal{P}_{y}^{(j)}+\hat{\mathbf{e}}_{z} \mathcal{P}_{z}^{(j)}$, such that $\mathcal{P}_{x}^{(j)}=\left(\mathcal{P}_{-1}^{(j)}-\mathcal{P}_{+1}^{(j)}\right) / \sqrt{2}$ and $\mathcal{P}_{y}^{(j)}=-i\left(\mathcal{P}_{-1}^{(j)}+\mathcal{P}_{+1}^{(j)}\right) / \sqrt{2}$. We obtain

$$
\begin{aligned}
& \dot{\mathcal{P}}_{x}^{(j)}=\left(i \Delta_{0}-i \tilde{\delta}-\gamma\right) \mathcal{P}_{x}^{(j)}-\bar{\delta} \mathcal{P}_{y}^{(j)}, \\
& \dot{\mathcal{P}}_{y}^{(j)}=\left(i \Delta_{0}-i \tilde{\delta}-\gamma\right) \mathcal{P}_{y}^{(j)}+\bar{\delta} \mathcal{P}_{x}^{(j)}+i \xi \epsilon_{0} \mathcal{E}_{0} / \mathcal{D},
\end{aligned}
$$

where $\tilde{\delta}=\left(\delta_{+}^{z}-\delta_{-}^{z}\right) / 2, \bar{\delta}=\left(\delta_{+}^{z}+\delta_{-}^{z}\right) / 2$, and $\Delta_{0}$ denotes the detuning of the $m=0$ state. The incident light directly drives only $\mathcal{P}_{y}^{(j)}$, but the energy splitting of the levels $|m= \pm 1\rangle$ introduces a coupling between $\mathcal{P}_{x}^{(j)}$ and $\mathcal{P}_{y}^{(j)}$. Although the incident field is perpendicular to $\mathcal{P}_{x}^{(j)}$, the light can therefore still excite $\mathcal{P}_{x}^{(j)}$ by first driving $\mathcal{P}_{y}^{(j)}$. The $J=0 \rightarrow J^{\prime}=1$ transition is isotropic when the excited-state energies are degenerate and any orientation of the orthogonal basis also forms an eigenbasis. For $\bar{\delta} \neq 0, \mathcal{P}_{x / y}^{(j)}$ no longer are eigenstates. The dipoles are consequently turned toward the $x$ axis by the rotation around the effective magnetic field.

For the entire interacting many-body system we numerically calculate the optical response for different Zeeman shifts and lattice heights and show in Fig. 2(a) the dynamics of the total polarization of the system $\left|\mathcal{P}^{\text {tot }}\right|=\left|\sum_{j, k} \mathcal{P}_{k}^{(j)} \hat{\mathbf{e}}_{k}\right| / N$ [in all the numerical results, 
the polarization amplitudes are expressed in the dimensionless form $\left.\mathcal{P} \rightarrow \mathcal{D P} k^{3} /\left(6 \pi \epsilon_{0} \mathcal{E}_{0}\right)\right]$. The incident light excites the $y$ components of the atomic dipoles. Analogously to the single atom case, the Zeeman shifts turn the polarization density toward the $x$ direction. At the resonance [36] $\left(\delta_{+}^{z}, \delta_{-}^{z}, \Delta_{0}\right)=(1.1,1.1,0.65) \gamma$ we find the dipoles almost entirely along the $x$ direction 43 . After the evolution has reached the steady state, the Zeeman shifts and the incident laser are turned off, resulting in a decay of the excitations. We fit the exponential functions to the decay profiles to obtain numerical estimates for the collective radiative linewidths that we later compare with the collective eigenvalues. For $\delta_{ \pm}^{z}=0$, the dipoles are in the lattice plane and the radiative decay rate $0.79 \gamma$ is close to the single atom linewidth. However, for $\left(\delta_{+}^{z}, \delta_{-}^{z}, \Delta_{0}\right)=(1.1,1.1,0.65) \gamma$ we find strongly suppressed decay of $0.14 \gamma$, indicating that the entire collective radiative excitation is dominated by subradiance. This is very different from the observation of long tails of radiative decay where only an extremely small fraction of the total excitation exhibits enhanced lifetime [29].

The lattice confinement affects the subradiant decay [Fig. 2(b)] and for more strongly fluctuating atomic positions we obtain faster decay rates with $0.18 \gamma$ and $0.28 \gamma$ for $s=20$ and $s=5$, respectively. For the case of fixed atomic positions a better fit is obtained by a double exponential (reflecting the occupation of eigenmodes with different linewidths, as explained later) $b_{1} e^{-c_{1} t}+b_{2} e^{-c_{2} t}$, with $b_{1} \simeq 0.72, c_{1} \simeq 0.0032 \gamma, b_{2} \simeq 0.24, c_{2} \simeq 0.027 \gamma$. The decay is dominated by an exponent that is about 300 times smaller than the one for a single atom.

Owing to the resonant DD interactions the atoms respond collectively to light, exhibiting collective excitation eigenmodes with distinct collective radiative linewidths and line shifts. We can qualitatively understand the response by analyzing the behavior of the most dominant modes. The incident light is phase-matched to a smoothly-varying, phase-coherent excitation of the atoms. The linear polarization couples to a collective ("coherent in-plane") mode in which all the dipoles are coherently oscillating along the $y$ direction with the excitation $\mathcal{P}_{I}$-a collective eigenmode of the system in the absence of the Zeeman shifts. Since all the dipoles in this mode are in the lattice plane, $\mathcal{P}_{I}$ is responsible for strong reflection and transmission of light. For nonzero Zeeman shifts the mode no longer is an eigenmode, but as in the single atom case, the polarization of the atoms is then turned toward the $x$ axis. This reorientation can be qualitatively analyzed by a simple two-mode model when we assume that $\mathcal{P}_{I}$ is predominantly coupled with a phasecoherent collective ("coherent perpendicular") excitation $\mathcal{P}_{P}$ where all the atomic dipoles are oscillating in phase, normal to the plane 36. Also this mode is a collective eigenmode for $\delta_{ \pm}^{z}=0$. We can now establish an effective two-mode dynamics 36

$$
\begin{aligned}
\dot{\mathcal{P}}_{P} & =\left(i \Delta_{P}-i \tilde{\delta}-v_{P}\right) \mathcal{P}_{P}-\bar{\delta} \mathcal{P}_{I}, \\
\dot{\mathcal{P}}_{I} & =\left(i \Delta_{I}-i \tilde{\delta}-v_{I}\right) \mathcal{P}_{I}+\bar{\delta} \mathcal{P}_{P}+i \xi \epsilon_{0} \mathcal{E}_{0} / \mathcal{D},
\end{aligned}
$$

where $v_{P / I}$ are the collective linewidths of the corresponding eigenmodes of the many-atom system (for $\left.\delta_{ \pm}^{z}=0\right)$ and $\Delta_{P / I}=\omega-\omega_{P / I}=\Delta_{0}+\delta_{P / I}$ are the detunings of the incident light from the resonances of these modes (that are shifted by $\delta_{P / I}$ ).

The excitation $\mathcal{P}_{P}$ dominantly radiates within the plane, enhancing interactions between the atoms: For light to escape, it generally undergoes many scattering events, so that the collective mode becomes strongly subradiant. After the excitation is driven into $\mathcal{P}_{P}$, we set $\mathcal{E}_{0}=\bar{\delta}=0$, and the decay becomes slow. The light can be released by applying a fast $\pi$-Rabi-pulse using $\bar{\delta} \neq 0$ that transfers the excitation back to $\mathcal{P}_{I}$.

We also calculate the eigenmodes when $\delta_{ \pm}^{z}=0$ for the full interacting system of atoms and light, and analyze the occupations of the different eigenmodes in the steady-state responses of Fig. 2(b) (at $\gamma t=20)$. We use the occupation measure $L_{j}=\left|\mathrm{v}_{j}^{T} \mathrm{~b}\right|^{2} / \sum_{i}\left|\mathrm{v}_{i}^{T} \mathrm{~b}\right|^{2}$ for the eigenvector $\mathrm{v}_{j}$ in the state $\mathrm{b}$. The resonance linewidths are then compared with the calculated decay rates of Fig. 2(b). We find that the steady-state excitation of the $\delta_{ \pm}^{z}=0$ fixed atomic position case is dominated by the collective $\mathcal{P}_{I}$ excitation eigenmode with about $50 \%$ of the total excitation [36. Its linewidth $v_{I} \simeq 0.79 \gamma$ almost perfectly matches with the fitted decay rate $0.80 \gamma$ in Fig. 2(b). For the $\left(\delta_{+}^{z}, \delta_{-}^{z}, \Delta_{0}\right)=(1.1,1.1,0.65) \gamma$ fixed atomic position case the fitting of the radiative decay to a double-exponential in Fig. 2(b) provided a much better result. This slowly-decaying case is dominated by the subradiant $\mathcal{P}_{P}$ excitation eigenmode with about $70 \%$ of the total excitation [36. The linewidth $v_{P} \simeq 3.1 \times 10^{-3} \gamma$ indicates a strongly subradiant excitation and again very closely matches with the dominant exponent $3.2 \times 10^{-3} \gamma$ of the decay in Fig. 2(b). The reason for the doubleexponential decay in Fig. 2(b) is a prominent excitation $\sim 15 \%$ of an additional eigenmode whose linewidth $\simeq 0.015 \gamma$ notably differs from that of $\mathcal{P}_{P}$.

Although the subradiant eigenmode with $v_{P} \simeq 3.1 \times$ $10^{-3} \gamma$ has a uniform phase profile, its amplitude is smaller close to the lattice edges [36]. This suggests that even a more targeted excitation of this mode can be achieved using a focused Gaussian laser beam. Indeed, a Gaussian beam with the standard deviation $6 a$ increases the occupation to $98 \%$ of the total excitation [36]. The corresponding dynamics provides an excellent fit to a single exponential with a decay rate of $3.1 \times 10^{-3} \gamma$.

The many-body nature of the light-mediated interactions manifests itself in a strong dependence of the suppressed decay on the size of the system. In Fig. 3(b) we show the linewidth $v_{P}$ as a function of the atom number $N$. For fixed atomic positions the mode be- 
comes increasingly more subradiant in larger lattices with $v_{P} / \gamma \simeq N^{-0.91}$. The fluctuations of the atomic positions suppress the linewidth narrowing and, e.g., $s=50$ has the large array limit $v_{P} \simeq 0.15 \gamma$. Using tight confinement in the Lamb-Dicke regime $\ell_{j} \ll a$, e.g., by optical tweezers, can significantly increase the lifetime of the subradiant state in large systems.

By varying the lattice spacing for different atom numbers we find that $v_{P}$ has a minimum around $a / \lambda=0.7-0.8$ [Fig. 3(a)]. Around the minimum $v_{P}$ is also the most subradiant linewidth of the system. The engineered excitations have particularly narrow linewidths for far reddetuned optical lattices for which $a / \lambda \gtrsim 0.55$.

The narrow linewidth $v_{P}$ manifests itself also in the resonance of the scattered light (Fig. 4). We display the spectrum of the steady-state response of the forward scattered light into a narrow cone of $|\sin \theta| \lesssim 0.1$. The full numerical simulation is compared with the two-mode model of Eqs. (4) that qualitatively captures the main features of the spectra, indicating that the resonance behavior is dominated by the two collective modes. The spectra exhibit a Fano resonance due to a destructive interference between different scattering paths that involve either the excitation $\mathcal{P}_{I}$ only, or a scattering via $\mathcal{P}_{P}$, as in $\mathcal{P}_{I} \rightarrow \mathcal{P}_{P} \rightarrow \mathcal{P}_{I}$. One can see from Eqs. (4) [36] that the forward or back scattered light is suppressed when $\bar{\delta}^{2} \gg v_{P} v_{I}$ and that the resonances correspond to high (low) occupations of $\mathcal{P}_{P}\left(\mathcal{P}_{I}\right)$ excitations. In the limit that $\mathcal{P}_{P}$ is not strongly driven, the narrow spectral resonance is a direct consequence of its subradiant linewidth in a large lattice (the resonances strongly depend on the lattice size; Fig. (4), and the interference is analogous to the interference of bright and dark modes in the electromagnetically-induced transparency (EIT) 44]. If $\mathcal{P}_{P}$ is strongly excited by the Zeeman shifts, the resonance notably broadens and its width can be approximated by $\left[\left(v_{I}^{2}+4 \bar{\delta}^{2}\right)^{1 / 2}-v_{I}\right] / 2\left(\right.$ for $\left.v_{P} / \bar{\delta} \simeq 0\right)[36$. In the limit of a large lattice the optical response varies between a full transmission $\left(\mathcal{P}_{P}\right.$ resonance) and complete reflection $(\bar{\delta}=0)[36$. Narrow transmission resonances due to collective radiative interference may also be achieved in magnetodielectric solid-state resonator systems [45], and EIT in an optical lattice has been proposed [32.

In conclusion, we showed that collective light-atom interactions can be harnessed for a controlled preparation of a single, spatially-extended, multiatom subradiant excitation eigenmode, storing the incident light. The possibility to engineer optical interactions may be promising, e.g., for the control of many-atom light shifts in lattice clocks [1, 2], and our subradiant state exhibits suppressed shifts [36]. Moreover, the narrow resonance features are very sensitive to the Zeeman shifts and could also provide a detection mechanism of weak magnetic fields [36. Unlike in a magnetometry using EIT 46 in weakly interacting vapors, the width of the resonance here is not limited by the single atom linewidth, but by the much narrower

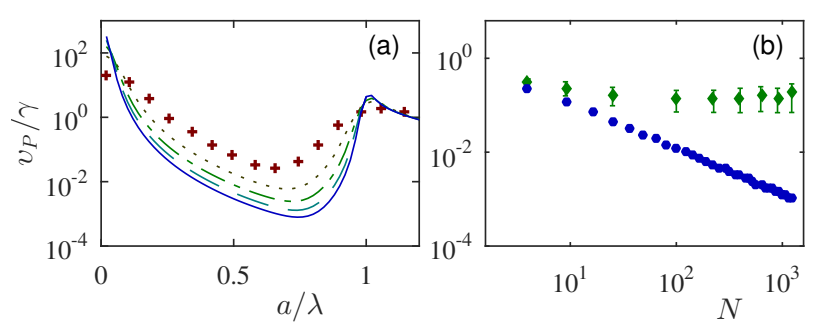

FIG. 3. The resonance linewidth $v_{P}$ of the subradiant collective eigenmode where the atomic dipoles coherently point to the normal of the lattice. (a) The dependence on the lattice spacing $a$. The curves from top: arrays $5 \times 5,10 \times 10,15 \times 15$, $20 \times 20,25 \times 25$. (b) The dependence on the number of atoms for $a=0.55 \lambda: s=50$ (top curve), the atoms at fixed positions (lower curve). For instance, $v_{P} \simeq 1.0 \times 10^{-3} \gamma$ for the $35 \times 35$ lattice in the lower curve. In comparison, the most subradiant eigenmode in this case has a linewidth $1.5 \times 10^{-4} \gamma$.
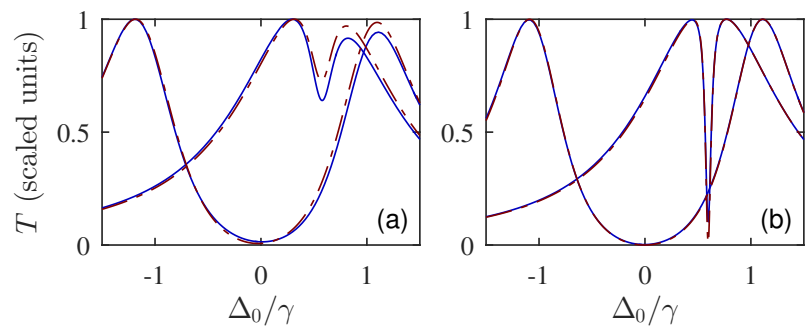

FIG. 4. The spectrum of forward or back scattered light and Fano resonances for different orientations of the dipoles: $\left(\delta_{+}^{z}, \delta_{-}^{z}\right)=(0.1,0.2) \gamma$ (the orientation not far from the lattice plane; narrow resonances), $(0.45,1.75) \gamma$ (the orientation approximately normal to the plane; broad resonances) for a lattice of fixed atomic positions and (a) $3 \times 3$; (b) $20 \times 20$ sites. Numerical simulation (blue, solid curves), two-mode model (red, dashed curves) with numerically calculated eigenvalues for the two dominant eigenmodes $\left(\delta_{P}+i v_{P}\right) / \gamma \simeq-0.65+$ $0.0031 i(20 \times 20),-0.62+0.12 i(3 \times 3)$, and $\left(\delta_{I}+i v_{I}\right) / \gamma \simeq$ $-0.68+0.79 i(20 \times 20),-0.59+0.83 i(3 \times 3)$. The scattering resonance is approximately at the effective resonance of the subradiant mode [Eq. 4a] ] $\Delta_{P}-\tilde{\delta} \simeq 0$.

collective subradiant linewidth, resulting, e.g., in a sharp dispersion at the transmission resonance (Fig. 4) and a large group delay.

We acknowledge financial support from the EPSRC and the use of the IRIDIS High Performance Computing Facility at the University of Southampton.

[1] T. L. Nicholson, S. L. Campbell, R. B. Hutson, G. E. Marti, B. J. Bloom, R. L. McNally, W. Zhang, M. D. Barrett, M. S. Safronova, G. F. Strouse, W. L. Tew, and J. Ye, Nat Commun 6, 6896 (2015).

[2] S. L. Bromley, B. Zhu, M. Bishof, X. Zhang, T. Bothwell, J. Schachenmayer, T. L. Nicholson, R. Kaiser, S. F. Yelin, 
M. D. Lukin, A. M. Rey, and J. Ye, Nat Commun 7, 11039 (2016)

[3] D. Budker and M. Romalis, Nature Physics 3, 227 (2007).

[4] K. Hammerer, A. S. Sørensen, and E. S. Polzik, Rev. Mod. Phys. 82, 1041 (2010)

[5] C. Liu, Z. Dutton, C. H. Behroozi, and L. H. Hau, Nature 409, 490 (2001).

[6] M. Fleischhauer and M. D. Lukin, Phys. Rev. Lett. 84, 5094 (2000).

[7] T. Chanelière, D. N. Matsukevich, S. D. Jenkins, S.-Y. Lan, T. A. B. Kennedy, and A. Kuzmich, Nature 438, 833 (2005).

[8] K. S. Choi, H. Deng, J. Laurat, and H. J. Kimble, Nature 452, 67 (2008).

[9] D. Tong, S. M. Farooqi, J. Stanojevic, S. Krishnan, Y. P. Zhang, R. Côté, E. E. Eyler, and P. L. Gould, Phys. Rev. Lett. 93, 063001 (2004).

[10] R. Heidemann, U. Raitzsch, V. Bendkowsky, B. Butscher, R. Löw, L. Santos, and T. Pfau, Phys. Rev. Lett. 99, 163601 (2007).

[11] J. D. Pritchard, D. Maxwell, A. Gauguet, K. J. Weatherill, M. P. A. Jones, and C. S. Adams, Phys. Rev. Lett. 105, 193603 (2010).

[12] T. Wilk, A. Gaëtan, C. Evellin, J. Wolters, Y. Miroshnychenko, P. Grangier, and A. Browaeys, Phys. Rev. Lett. 104, 010502 (2010).

[13] H. Schempp, G. Günter, C. S. Hofmann, C. Giese, S. D. Saliba, B. D. DePaola, T. Amthor, M. Weidemüller, S. Sevinçli, and T. Pohl, Phys. Rev. Lett. 104, 173602 (2010).

[14] R. H. Dicke, Phys. Rev. 93, 99 (1954).

[15] M. Gross and S. Haroche, Phys. Rep. 93, 301 (1982).

[16] A. Goban, C.-L. Hung, J. D. Hood, S.-P. Yu, J. A. Muniz, O. Painter, and H. J. Kimble, Phys. Rev. Lett. 115, 063601 (2015)

[17] S. J. Roof, K. J. Kemp, M. D. Havey, and I. M. Sokolov, Phys. Rev. Lett. 117, 073003 (2016).

[18] M. O. Araújo, I. Krešić, R. Kaiser, and W. Guerin, Phys. Rev. Lett. 117, 073002 (2016)

[19] C. C. Kwong, T. Yang, M. S. Pramod, K. Pandey, D. Delande, R. Pierrat, and D. Wilkowski, Phys. Rev. Lett. 113, 223601 (2014)

[20] J. Keaveney, A. Sargsyan, U. Krohn, I. G. Hughes, D. Sarkisyan, and C. S. Adams, Phys. Rev. Lett. 108, 173601 (2012).

[21] Z. Meir, O. Schwartz, E. Shahmoon, D. Oron, and R. Ozeri, Phys. Rev. Lett. 113, 193002 (2014).

[22] R. Röhlsberger, K. Schlage, B. Sahoo, S. Couet, and R. Rüffer, Science 328, 1248 (2010).

[23] S. D. Jenkins, J. Ruostekoski, J. Javanainen, R. Bourgain, S. Jennewein, Y. R. P. Sortais, and A. Browaeys,
Phys. Rev. Lett. 116, 183601 (2016)

[24] S. Jennewein, M. Besbes, N. J. Schilder, S. D. Jenkins, C. Sauvan, J. Ruostekoski, J.-J. Greffet, Y. R. P. Sortais, and A. Browaeys, Phys. Rev. Lett. 116, 233601 (2016).

[25] R. G. DeVoe and R. G. Brewer, Phys. Rev. Lett. 76, 2049 (1996)

[26] C. Hettich, C. Schmitt, J. Zitzmann, S. Khn, I. Gerhardt, and V. Sandoghdar, Science 298, 385 (2002)

[27] B. H. McGuyer, M. McDonald, G. Z. Iwata, M. G. Tarallo, W. Skomorowski, R. Moszynski, and T. Zelevinsky, Nat. Phys. 11, 32 (2015).

[28] Y. Takasu, Y. Saito, Y. Takahashi, M. Borkowski, R. Ciuryło, and P. S. Julienne, Phys. Rev. Lett. 108, 173002 (2012)

[29] W. Guerin, M. O. Araújo, and R. Kaiser, Phys. Rev. Lett. 116, 083601 (2016)

[30] S. D. Jenkins and J. Ruostekoski, Phys. Rev. A 86, 031602(R) (2012).

[31] M. Antezza and Y. Castin, Phys. Rev. A 88, 033844 (2013)

[32] R. J. Bettles, S. A. Gardiner, and C. S. Adams, Phys. Rev. A 92, 063822 (2015).

[33] S.-M. Yoo and S. M. Paik, Opt. Express 24, 2156 (2016)

[34] S. V. Syzranov, M. L. Wall, B. Zhu, V. Gurarie, and A. M. Rey, "eprint arxiv:1512.08723," (2015).

[35] O. Morsch and M. Oberthaler, Rev. Mod. Phys. 78, 179 (2006).

[36] See Supplemental Material for technical details.

[37] F. Gerbier, A. Widera, S. Fölling, O. Mandel, and I. Bloch, Phys. Rev. A 73, 041602 (2006).

[38] J. Ruostekoski and J. Javanainen, Phys. Rev. A 55, 513 (1997).

[39] O. Morice, Y. Castin, and J. Dalibard, Phys. Rev. A 51, 3896 (1995).

[40] J. Javanainen, J. Ruostekoski, B. Vestergaard, and M. R. Francis, Phys. Rev. A 59, 649 (1999).

[41] M. D. Lee, S. D. Jenkins, and J. Ruostekoski, Phys. Rev. A 93, 063803 (2016)

[42] J. D. Jackson, Classical Electrodynamics, 3rd ed. (Wiley, New York, 1999).

[43] We found that the many-atom dynamics is practically identical when we utilize the symmetry of Eqs. (3) and (4b) and transform to any new set of parameters $\Delta_{0} \rightarrow \Delta_{0}+\Delta, \delta_{+}^{z} \rightarrow \delta_{+}^{z}+\Delta, \delta_{-}^{z} \rightarrow \delta_{-}^{z}-\Delta$, for some $\Delta$.

[44] M. Fleischhauer, A. Imamoglu, and J. P. Marangos, Rev. Mod. Phys. 77, 633 (2005).

[45] S. D. Jenkins and J. Ruostekoski, Phys. Rev. Lett. 111, 147401 (2013).

[46] M. Fleischhauer, A. B. Matsko, and M. O. Scully, Phys. Rev. A 62, 013808 (2000). 